\title{
Chlamydia trachomatis serotype A infections in the Amazon region of Brazil: prevalence, entry and dissemination
}

\author{
Marluísa de Oliveira Guimarães Ishak ${ }^{[1]}$, Maurimélia Mesquita Costa $a^{[1]}$, \\ Núbia Caroline Costa de Almeida ${ }^{[1]}$, Angélica Menezes Santiago ${ }^{[1]}$, \\ William Botelho de Brito[1], Antonio Carlos Rosário Vallinoto[ ${ }^{[1]}$, \\ Vânia Nakauth Azevedo ${ }^{[1]}$ and Ricardo Ishak ${ }^{[1]}$
}

[1]. Laboratório de Virologia, Instituto de Ciências Biológicas, Universidade Federal do Pará, Belém, Pará, Brasil.

\begin{abstract}
Introduction: Chlamydia infection is associated with debilitating human diseases including trachoma, pneumonia, coronary heart disease and urogenital diseases. Serotypes of C. trachomatis show a fair correlation with the group of diseases they cause, and their distribution follows a well-described geographic pattern. Serotype A, a trachoma-associated strain, is known for its limited dissemination in the Middle East and Northern Africa. However, knowledge on the spread of bacteria from the genus Chlamydia as well as the distribution of serotypes in Brazil is quite limited. Methods: Blood samples of 1,710 individuals from ten human population groups in the Amazon region of Brazil were examined for antibodies to Chlamydia using indirect immunofluorescence and microimmunofluorescence assays. Results: The prevalence of antibodies to Chlamydia ranged from $23.9 \%$ (Wayana-Apalai) to $90.7 \%$ (Awa-Guaja) with a mean prevalence of $50.2 \%$. Seroreactivity was detected to C. pneumoniae and to all serotypes of $C$. trachomatis tested; furthermore, we report clear evidence of the as-yet-undescribed occurrence of serotype A of C. trachomatis. Conclusions: Specific seroreactivity not only accounts for the large extent of dissemination of C. trachomatis in the Amazon region of Brazil but also shows an expanded area of occurrence of serotype A outside the epidemiological settings previously described. Furthermore, these data suggest possible routes of Chlamydia introduction into the Amazon region from the massive human migration that occurred during the 1,700s.
\end{abstract}

Keywords: Chlamydia trachomatis. Serotype A. Seroprevalence.

\section{INTRODUCTION}

The genus Chlamydia is represented by four species: Chlamydia trachomatis, Chlamydia pneumoniae, Chlamydia psittaci and Chlamydia pecorum ${ }^{(1)}$. With the exception of C. pecorum (an animal pathogen not associated with human infections), Chlamydia species exhibit important associations with human diseases and infections.

Chlamydia trachomatis encompasses 19 serotypes, among which serotypes D-K are largely associated with urogenital infections, such as non-gonococcal urethritis, and are responsible for complications including salpingitis, pelvic inflammatory disease, and infertility. In addition, serotypes $\mathrm{L}_{1}-\mathrm{L}_{3}$ are associated with lymphogranuloma venereum ${ }^{(1)(2)(3)(4)(5)}$. Infections with serotypes A-C are etiologically involved in trachoma and are geographically distributed within specific areas where the disease

Corresponding author: Dr. Ricardo Ishak. Laboratório de Virologia/ICB/UFPA. Rua Augusto Correa 01, Cidade Universitária Prof. José da Silveira Netto, Guamá, 66075-900 Belém, Pará, Brasil.

Phone/Fax: 5591 3201-7587

e-mail:rishak@ufpa.br

Received 20 January 2015

Accepted 26 March 2015 is endemic. Serotype A is known for its limited geographic distribution within the Middle East and Northern Africa; serotype $\mathrm{B}_{\mathrm{a}}$ is commonly found among the Amerindians of North America; and serotypes $\mathrm{B}$ and $\mathrm{C}$ are distributed worldwide ${ }^{(6)}$.

The present report is the first to describe the detection, occurrence, possible routes of entry, and dissemination of serotype A of $C$. trachomatis in geographical areas other than those previously reported.

\section{METHODS}

\section{Population groups and collection of samples}

Blood samples of 1,710 individuals from ten human population groups were examined for antibodies to Chlamydia. The included subjects comprised the following population groups: I) two groups from the City of Belém, Pará, Brazil including 99 patients at a university public hospital presenting symptoms of respiratory illnesses (e.g., asthma and chronic obstructive pulmonary disease; Hospital Universitário João de Barros Barreto) and 33 patients at a public hospital presenting symptoms of angina pectoris, recent acute myocardial infarction and non-recent myocardial infarction (Fundação Santa Casa de Misericórdia do Pará); II) 784 subjects from 
two major urban communities (Santarém and Castanhal) of the State of Pará, Brazil; III) 278 individuals from two isolated Afro-descendant communities located in Northern Brazil (Pacoval, State of Pará, and Curiau, State of Amapá) who originated from runaway individuals during the slavery period; IV) 194 individuals from a village originating from emigrants of North Africa (Mazagão, State of Amapá); and V) 313 subjects from three native Indian groups - the Awa-Guaja people (State of Maranhão), the Wayana-Apalai people and the Kokraimoro group (State of Pará).

All samples were randomly selected from previous investigations performed by the Virus Laboratory, which were designed to establish seroepidemiological frequencies of infectious agents in Amazonian human populations and provide diagnostic support for medical interventions when needed in urban, non-urban and Indian communities. Blood samples were collected by venopuncture to obtain plasma or serum, and specimens were stored at $-20^{\circ} \mathrm{C}$ until use.

\section{Ethical considerations}

The number of individuals examined in each community is listed in Table 1, and the approximate geographical distribution of the participants is shown in Figure 1. Participants signed an informed consent form agreeing to take part in the study, which was submitted and approved by the Ethics Committee of the Federal University of Pará.

\section{Indirect immunofluorescence assay}

Commercial slides (BioMerieux, Paris France) impregnated with serotype $\mathrm{L}_{2}$ of $C$. trachomatis were used for the screening of antibodies to Chlamydia, a serotype that cross reacts with all other serotypes of $C$. trachomatis as well as $C$. pneumoniae and C. psittaci(6) (7) (8). Technical details have been described previously ${ }^{(9)(10)}$. Samples were screened at a dilution of 1:32, and the positive samples were further tested at a dilution of 1:512 using an anti-immunoglobulin $\mathrm{G}$ (IgG) conjugate (BioMerieux, Paris, France). Samples exhibiting titers equal to or greater than 512 were tested with an anti-immunoglobulin M (IgM) conjugate (BioMerieux, Paris, France) to detect serological evidence of the persistence of the bacterium (i.e., IgG titers $\geq 512$ in the absence of $\operatorname{IgM})^{(11)(12)}$. Readings were performed with the aid of an Axiophot epiilumination microscope (Zeiss, Berlin, Germany).

\section{Microimmunofluorescence assay}

Chlamydia pneumoniae and the serotypes of $C$. trachomatis (A, B, B , C, D, E, F, G, H, I, J, K, L $\mathrm{L}_{1}, \mathrm{~L}_{3}$ ) were commercially obtained as elementary body suspensions grown in egg yolk from the Washington Research Foundation, Seattle, Washington, USA. The slides were prepared with 16 antigens at the Virus Laboratory [Instituto de Ciências Biológicas, Universidade Federal do Pará (ICB/UFPA)], as described by the manufacturer. Sterile egg yolk suspension was used as a control for non-specific reactions against the substrate and for fixing purposes.

The microimmunofluorescence (MIF) assay is a highly complex and fastidious test, and for some of the groups, only samples that elicited positive reactions with the indirect immunofluorescence assay (IFA) were tested using MIF. All of the 144 samples that tested positive from the two diseased groups, all of the samples from Mazagão, and approximately $13 \%(93 / 715)$ of randomly selected samples that tested positive with the IFA from other groups (with the aid of the EpiInfo software $)^{(13)}$ were serotyped using MIF. The samples were initially tested at a 1:8 dilution with phosphate-buffered saline (PBS) according to previously described methodology $y^{(14)(15)}$. Samples that tested positive were serially diluted (to 1:1,024) and tested against the reactive serotypes. For samples with serum

TABLE 1 - Population groups examined for the presence of antibodies to Chlamydia.

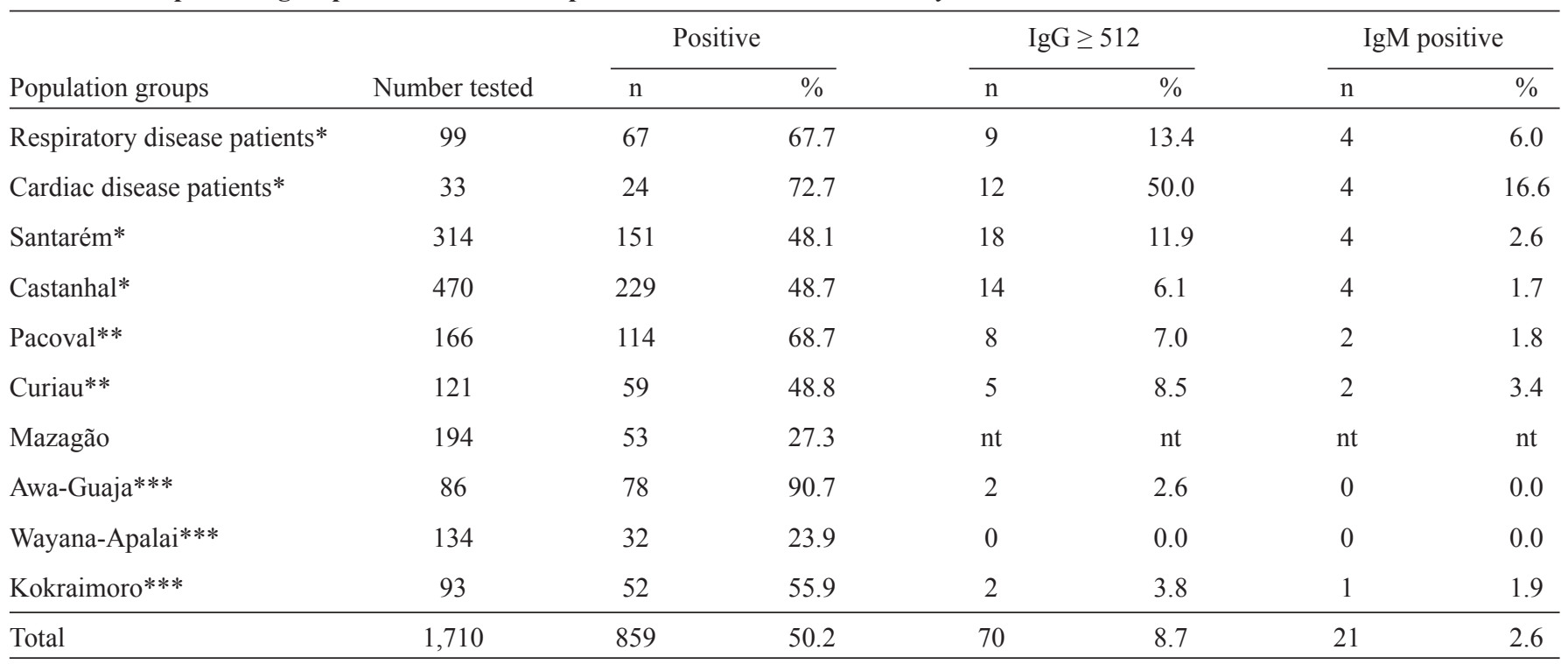

IgG: Immunoglobulin G; IgM: Immunoglobulin M; nt: not tested. *Urban groups; **Afro-descendant groups; ***Native Indian communities. 


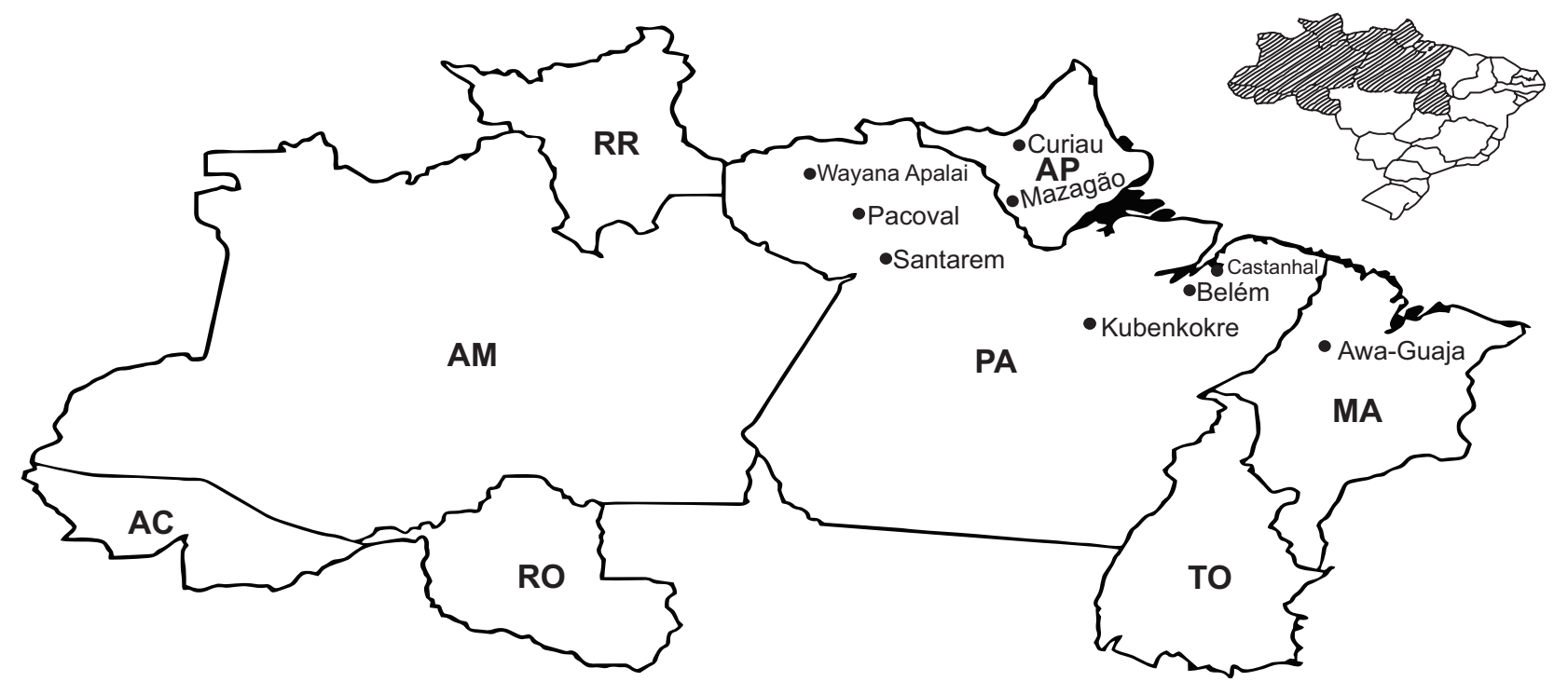

FIGURE 1 - Geographic distribution of population groups inhabiting the Amazon region of Brazil investigated for Chlamydia trachomatis infection. PA: Pará; AP: Amapá; MA: Maranhão; TO: Tocantins; AC: Acre; RO: Rondônia; AM: Amazonas; RR: Roraima.

reactivity to more than one serotype, titration is commonly performed because the serotype with the highest titer is assumed to have caused the most recent infection. A primary reaction was attributed to the antigenic serotype reacting to the highest titer. Differences of one-fold dilution between the serotype titers were not considered relevant and were considered as double or multiple past infections.

\section{RESULTS}

Table 1 depicts the prevalence of antibodies to Chlamydia. The prevalence of seroreactivity to Chlamydia ranged from $23.9 \%$ (Wayana-Apalai) to $90.7 \%$ (Awa-Guaja) with a mean prevalence of $50.2 \%$. IgG titers $\geq 512$ in the absence of IgM were found in $70 / 806(8.7 \%)$ of the positive subjects tested, indicating the possibility of bacterial persistence. IgM antibodies were present in $2.6 \%(21 / 870)$ of the subjects, suggesting an active infection with the bacterium. The presence of antibodies to Chlamydia in children and adolescents ( $0-15$ years of age) ranged from $30 \%$ (Castanhal) to $89.5 \%$ (Pacoval: data not shown).

The rates of seroreactivity to C. pneumoniae and to serotypes of $C$. trachomatis are shown in Table 2. Individual reactions were detected to all serotypes. Reactivity to serotype A was described in 15 samples of sera from six population groups (respiratory and cardiac patients from Belém; the general population of Castanhal, Pacoval, and Mazagão; and the Wayana Apalai Indian community). Among 15 samples that tested positive with IFA, reactivity to $C$. pneumoniae or to any of the C. trachomatis serotypes was not detected using the MIF assay in at least one serum sample from all population groups tested (with the sole exception of Mazagão).

\section{DISCUSSION}

The prevalence of antibodies to Chlamydia in both urban and non-urban communities in the Amazon region of Brazil has been described previously and is known to be largely variable $^{(9)(10)(15)}$. The dissemination of this bacterium is extensive and may play an important role in the perpetuation of infection in isolated, small native Indian communities where the risk of infertility is currently high ${ }^{(15)}$. The prevalence of Chlamydia antibodies reflects exposure to the bacterium; seroreactivity to both species predicts the possibility that a person may be experiencing disease either with or without a clinical or laboratory diagnosis. Clinical and laboratory differential diagnosis of chlamydial infections remains an uncommon practice, with the exception of trachoma.

Indeed, the presence of antibodies to Chlamydia at high titers $(\geq 512)$ in the absence of IgM (found in $8.7 \%$ of individuals) is a strong serological marker suggestive of persistence of the bacterium in the host. These characteristics identify persons acting as potential reservoirs for bacterial dissemination, with and without clinically apparent manifestations ${ }^{(16)}$. The use of nucleic acid amplification to detect persistent infections due to C. trachomatis has revealed prevalence rates ranging from $6.5 \%$ to $17.5 \%{ }^{(17)(18)}$, which is in agreement with the results described in the present paper and those of studies that performed isolation in cell cultures ${ }^{(10)}$ or serological methods ${ }^{(15)}$ to quantify persistent infections. Furthermore, antibodies to Chlamydia are widespread in children and adolescents in two of the communities tested, as well as in others described elsewhere in the Amazon region of Brazil ${ }^{(15)}$.

The application of serological methods for the detection of antibodies is an effective procedure to measure and define the 
TABLE 2 - Seroreactivity to Chlamydia trachomatis and Chlamydia pneumoniae based on MIF analysis.

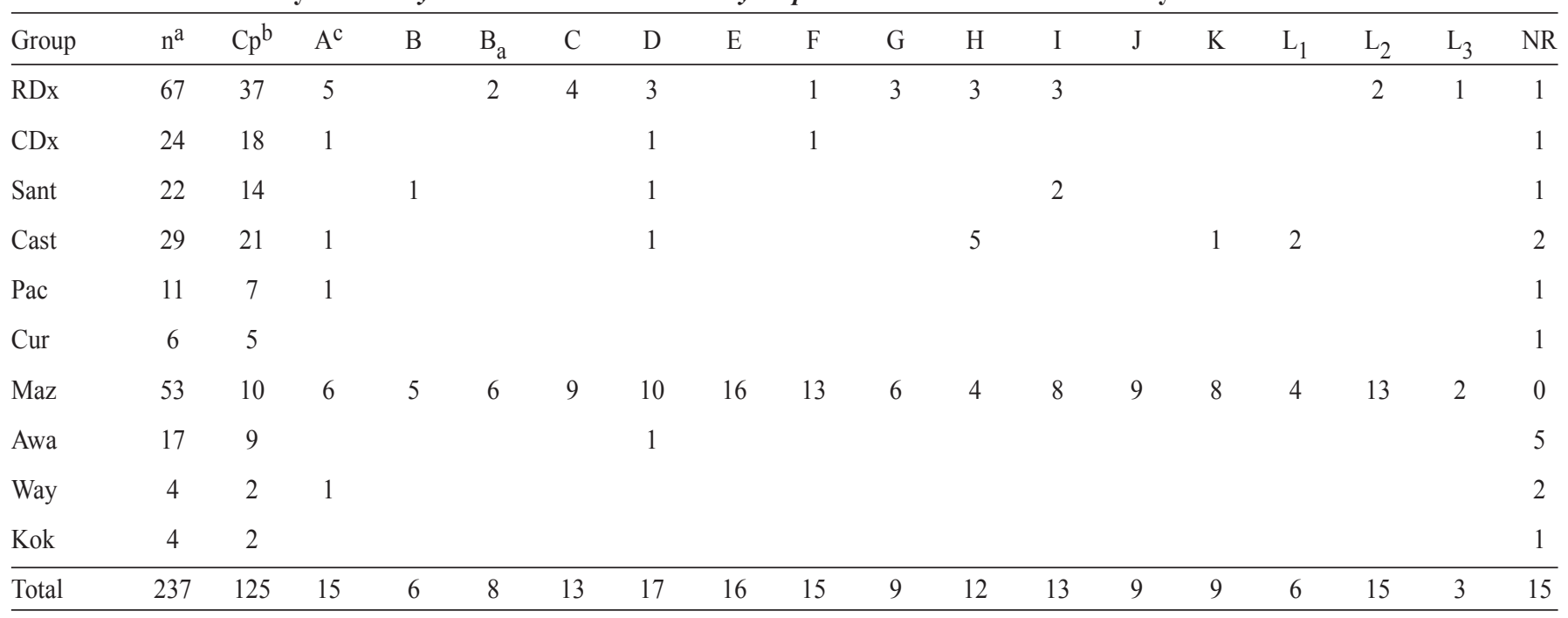

MIF: microimmunofluorescence; RDx: respiratory disease; CDx: cardiac disease; Sant: Santarém; Cast: Castanhal; Pac: Pacoval; Cur: Curiau; Maz: Mazagão; Awa: Awa-Guaja; Way: Wayana-Apalai; Kok: Kokraimoro; Cp: Chlamydia pneumoniae; NR: no reaction to both species of Chlamydia; - ${ }^{\mathrm{a}}$ number of samples tested; ${ }^{\mathrm{b}}$ C. pneumoniae); ${ }^{\mathrm{c}}$ serotypes of $C$. trachomatis, A to $\mathrm{L}_{3}$.

extent to which a population group has been previously exposed to an infectious agent, including Chlamydia infections, as well as determine whether the infection was caused by ocular or genital strains of $C$. trachomatis. Finally, these methods are useful for identifying the predominant serotypes distributed among a range of communities.

The combination of sequential seroreactions using the IFA followed by the MIF, although a complex technique, provides a reliable approach to obtain epidemiological information, such as infection with Chlamydia or its serotypes. Primary seroreactivity, using the MIF assay, has been used to detect serotypes related to sexual transmission of $C$. trachomatis and to serotypes A and $\mathrm{B}$, which are usually associated with ocular infections and the clinical presentation of trachoma in endemic areas (together with serotypes $\mathrm{B}_{\mathrm{a}}$ and C). Serotype A was recognized as a cause of trachoma within a limited geographic distribution in the Middle East and Northern Africa; serotypes B and C were identified as cosmopolitan serotypes; and serotype $\mathrm{B}_{\mathrm{a}}$ was found to be most prevalent among North American Indians ${ }^{()}$.

Serological evidence of human infection with serotype A of C. trachomatis is described in the present paper for the first time in the Amazon region of Brazil. We detected fifteen reactions to serotype A in six different groups tested; positive samples were detected among diseased subjects (both respiratory and cardiac illnesses) in Belém (the major urban community of the Amazon region of Brazil), in Castanhal (an urban area close to Belém), within one isolated Afro-descendant community (Pacoval), in one epidemiologically closed Indian community (Wayana-Apalai) and in Mazagão, a village that was established during the XVIII century from emigrants from North Africa. It is relevant to mention that two subjects showed single reactivity without secondary reactions to $C$. pneumoniae or other serotypes of $C$. trachomatis, and one of these subjects was a 12-year-old boy from Pacoval. Thus, this bacterium was found infecting persons with different ethnic backgrounds.

It is possible that serotype A was introduced into the Amazon region of Brazil during the massive human migration that occurred in the 1,700s. At that time, a group of more than 1,000 Portuguese Catholics were brought into the region from Northern Africa. These individuals were escaping Muslim persecution as a result of a religious war occurring in their hometown, a place in Northern Africa also called Mazagão. Since then, serotype A has spread to urban and non-urban populations. The only reactions to serotypes $\mathrm{E}$ and $\mathrm{J}$ of C. trachomatis were observed in residents of Mazagão.

The description of serotypes or genotypes of $C$. trachomatis is not a common procedure, and such analyses are typically focused on strains that are sexually transmitted; this is true for recent reports in $\mathrm{Brazil}^{(19)(20)}$. It is possible that the distribution of different serotypes over the years could have an important clinical impact, although these analyses are almost never performed for strains related to ocular infections.

The clinical and epidemiological observations reported by Freitas $^{(21)}$ indicate that the Amazon region of Brazil is endemic for trachoma, and has been for a while. However, the clinical cases may go undiagnosed or misdiagnosed. It is relevant to stress that during our medical expeditions, there was no detection of patients with ocular disease that resembled trachoma or conjunctivitis. However, the presence of trachoma has been associated with social conditions that favor the dissemination of Chlamydia by flies in Marajó Island, a part of the State of Pará ${ }^{(22)}$.

Additional evidence for the presence of $C$. pneumoniae among different population groups was reported using a method that enables the detection of specific reactions without the interference of cross reactions among the different species of Chlamy $\mathrm{dia}^{(23)(24)}$. Laboratory diagnosis of chlamydial infections 
is not commonly performed in the Amazon region, and it is expected that both C. trachomatis and C. pneumoniae are causative agents of clinical disease but are rarely considered for differential laboratory and clinical diagnosis.

Fifteen samples that were reactive to a genus-specific Chlamydia antigen with IFA did not show any reactivity using the MIF assay. It is possible that the IFA detected antibodies to a species of Chlamydia that was not used in the MIF assay, although the lack of reactivity suggests that $C$. psittaci or another species that has not yet been associated with human infections (such as C. pecorum) may be involved; this result requires further investigation.

The prevalence of Chlamydia in the Amazon region of Brazil indicates that both $C$. trachomatis and $C$. pneumoniae infect humans and thereby establish reservoirs to perpetuate these bacterial infections. It is also possible that species of Chlamydia currently unknown to man have crossed over from other animal species and now cause clinical and subclinical infections in humans in the Amazon region. Accurate clinical and laboratory diagnoses are rarely performed, and epidemiological information must be generated to improve the handling of potential chlamydial infections. In this study, the detection of serotype A of $C$. trachomatis was described for the first time in a geographical area that is different from its initial place of detection, and it is possible that this serotype was introduced with the emigrants from North Africa who settled in Mazagão. Detection of serotypes or genotypes of $C$. trachomatis causing ocular diseases as well as sexually transmitted disease must receive greater clinical and biological attention to eliminate these infections and their accompanying diseases.

\section{ACKNOWLEDGMENTS}

The authors acknowledge the participants in all of the communities examined as well as our working colleagues SEB Santos, JF Guerreiro, AKCR Santos, E Santos, V Monteiro, ES Abrahim and S Caniceiro.

\section{CONFLICT OF INTEREST}

The authors declare that there is no conflict of interest.

\section{FINANCIAL SUPPORT}

The present work was generously funded by the Programa Nacional de DST/AIDS, Ministry of Health, by the Conselho Nacional de Desenvolvimento Cientifico e Tecnológico (CNPq) and the Universidade Federal do Pará.

\section{REFERENCES}

1. Peeling W, Brunham RC. Chlamydiae as pathogens: new species and new issues. Emerg Infect Dis 1996; 2:1-16.

2. World Health Organization. Sexually transmitted diseases. WHO Press Release WHO/64; 1995.
3. Singh V, Salhan S, Das BC, Mittal A. Predominance of Chlamydia trachomatis serovars associated with urogenital infections in female in New Delhi, India. J Clin Microbiol 2003; 41:2700-2702.

4. Spaargaren J, Fennema HS, Morré SA, de Vries HJ, Coutinho RA. New lymphogranuloma venereum Chlamydia trachomatis variant, Amsterdam. Emerg Infect Dis 2005; 11:1090-1092.

5. Pathela P, Blank S, Schillinger JA. Lymphogranuloma venereum: old pathogen, new story. Curr Infect Dis Report 2007; 9:143-150.

6. Grayston JT, Wang SP. New knowledge of chlamydiae and the diseases they cause. J Infect Dis 1975; 132:87-105.

7. Campbell LA, Kuo CC, Grayston JT. Structural and antigenic analysis of Chlamydia pneumoniae. Infect Immun 1990; 58:93-97.

8. Treharne JD, Forsey T, Thomas BJ. Chlamydial serology. Br Med Bull 1983; 39:194-200.

9. Ishak MOG, Mumtaz G, Ishak R, Ridgway G. Prevalence of antibodies to Chlamydia trachomatis in population groups of Brazil, England and Portugal. Rev Inst Med Trop São Paulo 1988; 30:40-44.

10. Ishak MOG, Ishak R, Cruz AC, Santos DE, Salgado U. Chlamydial infection in the Amazon region of Brazil. Trans R Soc Trop Med Hyg 1993; 87:60-62.

11. Conway D, Glazener CM, Caul EO, Hodgson J, Hull MG, Clarke SK et al. Chlamydial serology in fertile women. Lancet 1984; 1:191-193.

12. Lobos T, Moreno RB, Saldias FP, Cartagena CS, Ferres MG. Seroprevalencia de infección por Chlamydia pneumoniae en población sana de Santiago de Chile. Rev Chil Enfermid Resp 1996; 12:145-150.

13. Centers for Disease Control and Prevention (CDC), World Health Organization (WHO). Software EpiInfo 6. version 6.04. A world processing, database and statistics program for public health. Atlanta, Geneve: CDC/WHO; 1996.

14. Wang SP, Grayston JT. Human serology in Chlamydia trachomatis infection with microimmunofluorescence. J Infect Dis 1974; 130:388-397.

15. Ishak MOG, Ishak R. O impacto da infecção por Chlamydia em populações indígenas da Amazônia brasileira. Cad Saude Publica 2001; 17:385-396.

16. Schachter J. Chlamydial infections. West J Med 1990; 153:523-534.

17. Lan J, Melgers I, Meijer CJLM, Walboomers JM, Roosendaal R, Burger $\mathrm{C}$ et al. Prevalence and serovar distribution of asymptomatic cervical Chlamydia trachomatis infections as determined by highly sensitive PCR. J Clin Microbiol 1995; 33:3194-3197.

18. Cheema MA, Rahman MU, Whitteem-Hudson JA, Hudson AP. Rapid communication: cervical Chlamydia trachomatis in women at low risk for infection. Am J Med Sci 2000; 319:123-125.

19. Lima HE, Oliveira MB, Valente BG, Afonso DA, Darocha WD, Souza MC, et al. Genotyping of Chlamydia trachomatis from endocervical specimens in Brazil. Sex Transm Dis 2007; 34:709-717.

20. Machado AC, Bandea CI, Alves MF, Joseph K, Igietseme J, Miranda $\mathrm{AE}$, et al. Distribution of Chlamydia trachomatis genovars among youths and adults in Brazil. J Med Microbiol 2011; 60:472-476.

21. Freitas CA. Prevalência do tracoma no Brasil. Rev Bras Malariol Doencas Trop 1976; 28:227-233.

22. Reilly LA, Favacho J, Garcez LM, Courtenay O. Preliminary evidence that synanthropic flies contribute to the transmission of trachoma-causing Chlamydia trachomatis in Latin America. Cad Saude Publica 2007; 23:1682-1688.

23. Grayston JT, Kuo CC, Campbell LA, Wang SP. Chlamydia pneumoniae sp. nov. for Chlamydia sp. TWAR. Int J Syst Evol Microbiol 1989; 39:88-90.

24. Ozanne G, Lefebvre J. Specificity of the microimmunofluorescence assay for the diagnosis of Chlamydia pneumoniae infections. Can J Microbiol 1992; 88:1185-1189. 